\title{
First Results with the Wealthy Garment ElectroCardiogram Monitoring System
}

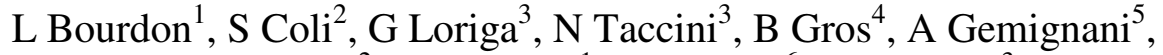 \\ D Cianflone ${ }^{2}$, F Chapotot ${ }^{1}$, A Dittmar $^{6}$, R Paradiso $^{3}$ \\ ${ }^{1}$ CRSSA, Grenoble, France \\ ${ }^{2}$ San Raffael Hospital, Milano, Italy \\ ${ }^{3}$ Smartex, Navacchio, Italy \\ ${ }^{4}$ CSEM, Neuchatel, Switzerland \\ ${ }^{5}$ University of Pisa, Pisa, Italy \\ ${ }^{6}$ INSA, Lyon, France
}

\begin{abstract}
The Wealthy project aimed at measuring physiological variables with sensors made with functionalised yarns integrated in a well fitting cloth. Two sets of experiments were carried out to verify whether the Wealthy garment provides ECG and respiratory data reliable and satisfactory for physicians. In the first one, ECG was simultaneously recorded by a standard device and the Wealthy system during simulation of common daily activity at the hospital in five male cardiac patients. No differences were consistently observed between standard and wealthy systems for visual and spectral analysis. The second set of experiment was performed in seven healthy subjects during either daily activities in normal or hot conditions $\left(45^{\circ} \mathrm{C}\right)$ or during the night. When the standard signal was rated as good, the Wealthy signal was rated as good or acceptable in $95 \%$ of time for ECG and $100 \%$ for the respiratory signal. Monitoring of ECG with fabric sensor is thus proved feasible.
\end{abstract}

\section{The WEALTHY system}

WEALTHY system is developed as the integration of several function modules. The main functions of the wearable modules [1] are shown in Figure 1, namely: sensing, conditioning, pre-processing, and data transmission. The garment interface is connected with the portable WEALTHY device (PPU), where the local processing as well as the communication with the network is performed. Most signals are transmitted unprocessed to the Monitoring System where they can be analyzed off-line at the monitoring center.

In order to offer full mobility to the user, signals are wirelessly transmitted from the PPU to the remote Monitoring System. The communication is based on a GPRS communication under TCP/IP protocol which can be replaced by a Bluetooth module in case of short range applications. All signals are sent in quasi real-time to the remote Monitoring Centre.

The conductive sensors (electrodes) have been realized with a yarn where two stainless steel wires are twisted around a viscose textile yarn (Elitè by Lineapiù SpA, Italy). Electrodes were knitted by using tubular intarsia technique to get a double face, where the external part is not conductive. The basal yarn (not sensitive) was the same yarn used as core for the conductive electrode yarn. To improve the electrical signal quality in dynamic condition a hydro-gel membrane could be added.

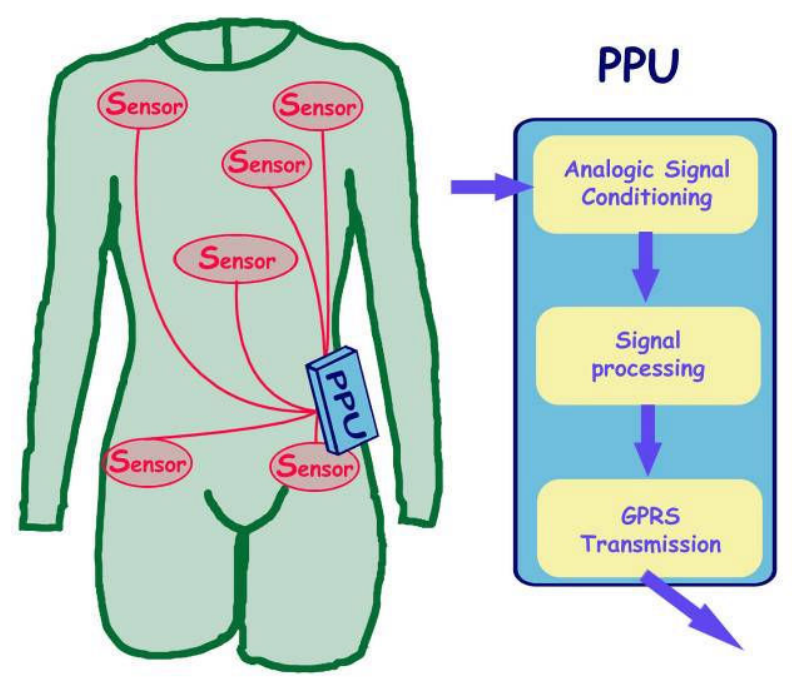

Figure 1: Overview of wearable WEALTHY's modules

Tracks have been realized by means of tubular intarsia technique. A supplementary layer has been woven by using of vanisé technique. The final tracks is a multi layered structure were the conductive surface is 
sandwiched between two insulated standard textile surfaces.

The respiratory activity is monitored using a impedance pneumography technic. This methodology need four electrodes placed on thoracic position [2]: the two outer ones are used to inject a high frequency current $(50 \mathrm{kHz})$ and the inner ones measure the voltage variation caused by thoracic impedance change. The output signal is modulated by changes in the body impedance accompanying the respiratory cycle.

Knitting fabric has been made with a flat-knitting machine (Vesta Vx 12 - Steiger). In figure 2 is shown the prototype model, electrodes and breathing sensors position.

Conductive yarns and fabrics are resistant to repeated washing in aqueous solutions, the washed electrodes can be used to detect ECG signal, the signals detected shown that the performances of the conductive fabric are not affected by the washing process.

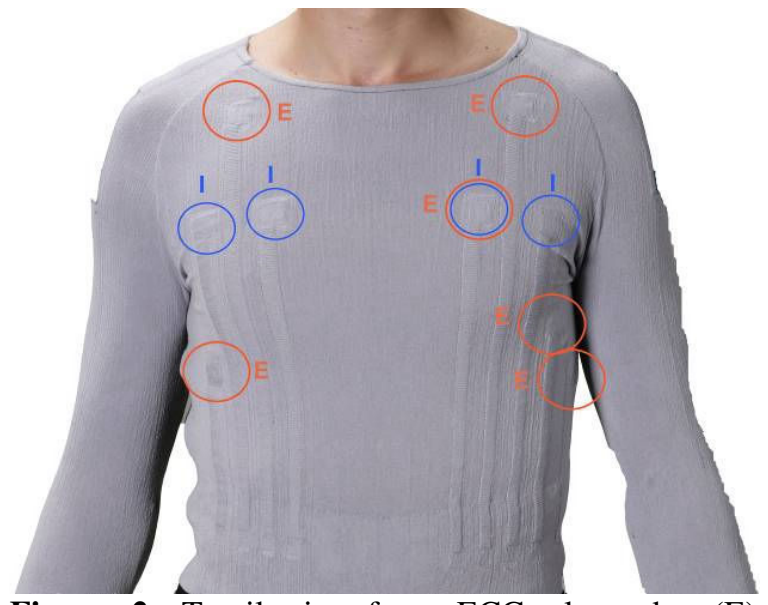

Figure 2: Textile interface: ECG electrodes (E), Impedance pneumography electrodes (I).

\section{Evaluation in cardiac patients}

the purpose was to verify that the ECG data generated by WEALTHY device are reliable and satisfactory for physicians, compared with those achievable with standard clinical devices and that the WEALTHY shirt wearability and comfort are satisfactory for the patient.

The validation phase was carried out at San Raffaele Hospital, Milano. Patient inclusion criteria are confirmed diagnosis of heart disease and stable clinical conditions, to allow at least light exercise and full cooperation during testing. A written informed consent is obtained by each patient according to the protocol approved by the local ethical committee. So far we have conducted tests on 5 male patients (median 54 years old, range 42-64).

In all patients the ECG was simultaneously recorded with the WEALTHY system (W) and our standard clinical radio-telemetry equipment (Mortara-Rangoni; R), under different conditions. The standard following ECG leads were recorded: DI, DII, V2 and V5. The WEALTHY shirt was prepared before each experiment by attaching hydrogel films on the inner surface of the tissue electrodes.

Patients were asked to follow a standard activity protocol lasting 35-45', in which the ECG was continuously recorded with alternative switching between leads. The protocol included bed rest, sitting, standing, light walking, etc. At the end of the test the patient were asked to provide an evaluation with an ad-hoc scale (ranging from 1 worst, to 5 best) on the comfort, the difficulty of wearing the shirt at the beginning of the test, and last their personal commitment to the possibility of using the garment for cardiac monitoring in the future.

Both quantitative and qualitative approaches have been used to analyse ECG data. For the first one, the ECG lead II signal obtained by both systems over a time period of 4 minutes, in a resting state, was treated using standard Power Spectral Density (PSD) analysis in order to compare the frequency distribution. This approach allows to identify differences in the ECG signal related to electrode and acquisition-system characteristics peculiarity. A qualitative approach has been used to specifically evaluate the clinical interpretability/quality of the ECG recordings obtained with both systems. For this analysis, five representative consecutive beats were selected for each lead and for the rest, light activity, and exercise phases of the protocol. A score of interpretability/quality ( 0 worst: not interpretable; to 4 best, fully interpretable, free of artefact) was used to provide a global score based on a judgement of the quality of the ECG and an analytical score for each ECG component ( $\mathrm{P}$ wave, PR tract, QRS complex, ST tract, T wave) analysed separately, then averaged on the 5 consecutive beats.

The PSD analysis performed on R and W ECG signals is nearly identical and the main frequency components are the same in three patients. In two patients, however, it shows differences in frequency distribution between ECGs systems, related to higher noise in standard ECG signal with respect to Wealthy one in the first one, and due to the unavoidable different lead positioning in limb leads between $\mathrm{R}$ and $\mathrm{W}$ systems in the other one.

In qualitative analysis, the average global score was 2.8 and 2.9 , respectively with $\mathrm{W}$ and $\mathrm{R}$ equipment. The average analytical score for the various ECG components, presented figure 3 , shows that WEALTHY compares well to standard clinical equipment independent of the ECG wave considered. Physical activity deteriorates ECG quality according to its intensity with both systems; however, WEALTHY does not seem to be inferior to 
clinical telemetry.

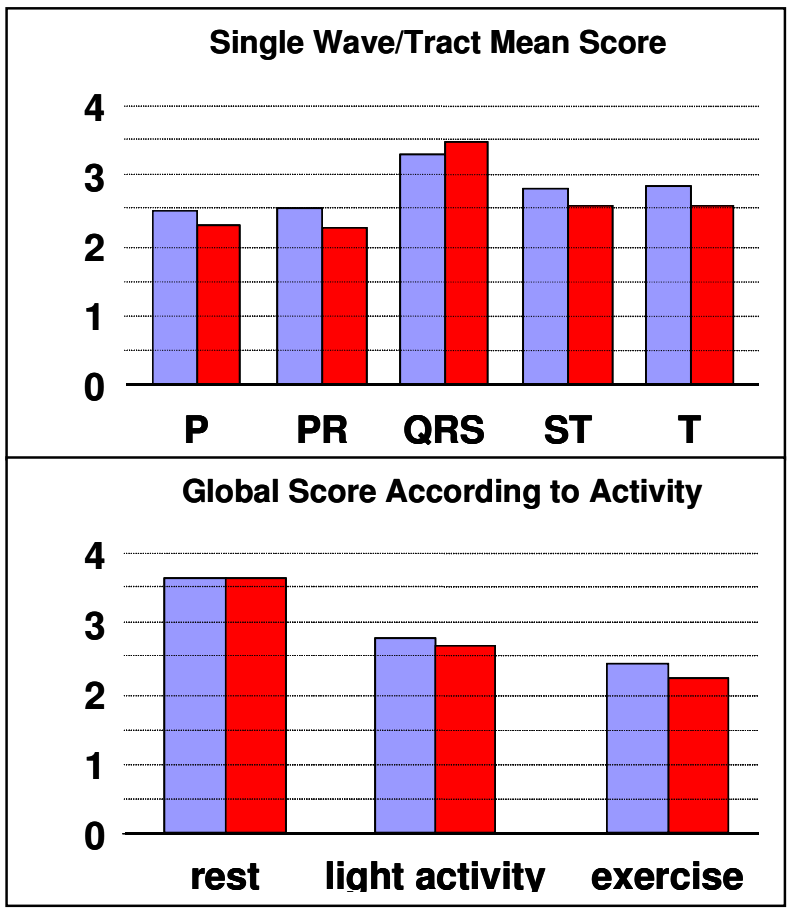

Figure 3: top : analytical score, average of 4 leads in 5 patients in 3 testing conditions. Bottom : global score according to activity. In both: Standard system in blue, and Wealthy system in red.

The use of the WEALTHY shirt has generally been well accepted, with nearly all patients reporting good comfort and no troubles in wearing the shirt at the beginning of the study.

In the experiments conducted so far, the performance of the WEALTHY shirt seems to compare well with that of standard ECG telemetry equipment. Quantitative analysis shows near identity between the rest tracings obtained with the two systems in 3 out of 5 patients while in the remaining 2 patients differences are not due to major problems in WEALTHYsystem.

Qualitative analysis demonstrates that, for clinical purposes (i.e. to be interpretable by a physician), ECG quality appears to be very close between the systems. ECG signal degrades with both systems during physical activity, but WEALTHY does not seem to be inferior compared to clinical telemetry in this setting.

The wide range of WEALTHY performance observed among different leads and different patients is at least partially due to different body constitutions; this fact points to the need for the future of at least a few different sizes of the shirt. Nevertheless D2 showed a good performance basically across all patients.
Patient tolerance does not seem a problem, at least for limited time use, as all cardiac patients found the shirt comfortable during the study.

\section{Evaluation in healthy subjects}

The aim was to compare the performances of WEALTHY System with respect to a standard portable device in the recording of ECG and Respiration signals on healthy subjects engaged in simulated working activity in the lab or during real nights.

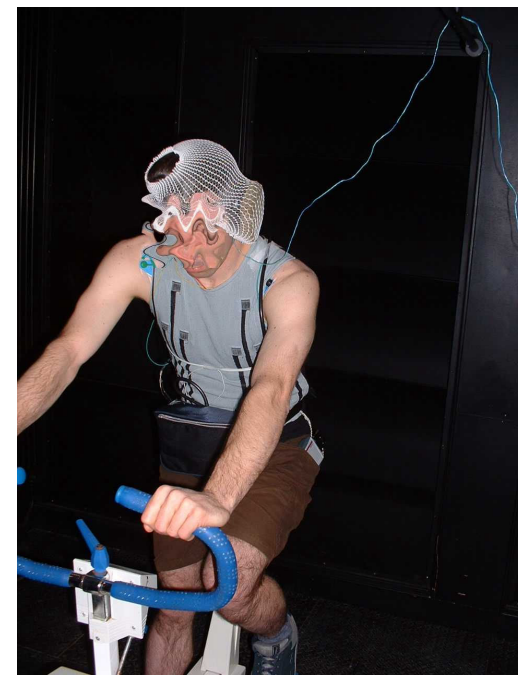

figure 4: subject exercising on an ergocycle into the climatic chamber set at $45^{\circ} \mathrm{C} \mathrm{Tdb}, 60 \%$ relative humidity.

Seven (7) male subjects volunteered to participate in the study $(31 \pm 5.6 \mathrm{yrs}$ old, $68 \pm 6.6 \mathrm{~kg}$ body weight, and $175.7 \pm 5.4 \mathrm{~cm}$ height). All acquisitions were carried out with the WEALTHY System and the Vitaport III® (Temec Instrument bv, the Netherland) as reference polygraphic recorder. Data acquisition characteristics were the same for both systems, i.e. ECG on five leads (DI, DII, DIII, V2, V5) at $128 \mathrm{~Hz}$ and respiration movements by standard resistive plethysmography, for the standard system, and by impedancemetry for the WEALTHY system, at $16 \mathrm{~Hz}$. Clock of both systems were synchronized just before the recordings to allow the comparison of the signals.

For every recordings, both systems were set in order to obtain the best signal during the preparation phase, thereafter no attempt to ameliorate the signal was done whatever its quality was along the "recording period", i.e. the period during which comparison between signals was performed. Three different experimental protocols were used: short acquisition (45 to $90 \mathrm{~min}$ ) in a controlled normal conditions $(\mathrm{CN})$ during resting conditions or during light exercise; short acquisition (45 to $90 \mathrm{~min}$ ) in a controlled harsh conditions $(\mathrm{CH})$ during a mix of resting 
and cycling periods (figure 5) performed into a climatic chamber set as $45{ }^{\circ} \mathrm{C} \mathrm{T}_{\mathrm{db}} / 60 \%$ rh (the test ended when tympanique temperature of the subject reached $38.5^{\circ} \mathrm{C}$ or after $60 \mathrm{~min}$ of exposure); and long acquisition (5 to 7 hours) in a "natural" setting (NS) during a normal sleeping night at home.

For each experiment, reference (R) and WEALTHY (W) signals were digitalized and stored in binary files in real time. Binary files were then off-line visualized with the Prana Viewer ${ }^{\circledR}$ (Phitools sarl, France) and quoted according to whether the signal allowed the determination of the heart/respiratory rate or better. ECG signal was quoted 1 (not interpretable) to 4 (good signal). RESP signal was considered as not interpretable (quoted 1), poorly interpretable (allowing just the calculation of the respiratory frequency, quoted 2) and good (quoted 4) when it would have allowed an estimation of the respiratory volume. Each recording was sliced in 15-sec long epochs; quotation was then made separately on each epoch. This procedure leads to a vector of values comprised between 1 and 4 for every experiment. $\mathrm{R}$ and $\mathrm{W}$ vectors from same experiment were then synchronized and data statistically analysed using Excel® (Microsoft Corporation).

About $31 \mathrm{~h}$ recordings were analysed. The first result is that any movement induces noise which make the signal poorly or not interpretable whatever the device used to record it. However, when movements are reduced or absent, raw signal is generally good for both traces, ECG and RESP.

A total of 5147 epochs (21h $26 \mathrm{~min} 45 \mathrm{sec}$ ) were quoted. Both $\mathrm{R}$ and $\mathrm{W}$ signals were rated the same level of quality $76.8 \pm 3.2 \%$ of time for ECG and $80.2 \pm 1 \%$ of time for RESP signal.

When the $\mathrm{R}$ signals were rated good (level 4) the $\mathrm{W}$ signal was rated acceptable or good (3 and 4 for ECG and 2 and 4 for RESP) $87.8 \%$ of time for the ECG signal and $92.2 \%$ of time for the RESP signal. The R signal was considered better than the $\mathrm{W}$ signal $18.2 \pm 5.2 \%$ of time for the ECG signal and $13.1 \pm 1.9$ of time for the RESP signal and the $\mathrm{W}$ signal was considered better than the $\mathrm{R}$ signal $4.8 \pm 1.0 \%$ of time for the ECG signal and $7.2 \pm$ $1.0 \%$ of time for the RESP signal.

There was no reported problems due to discomfort related to hydrogel membrane whatever the length of the recording. Moreover, $\mathrm{CH}$ tests were performed without using the hydrogel membrane; we consistently observed that both ECG and RESP W signals improve during the test while it was going worse for the $\mathrm{R}$ ones. As a consequence, $\mathrm{W}$ signal is more often better than $\mathrm{R}$ signal during this test than in other ones. This effect was probably due to the sweat heavily produced during the test which tends to decrease electrical impedance between the skin and the WEALTHY electrodes while it tends to unstuck tapped electrodes used for the Reference signal.

\section{General comments and conclusion}

1) In healthy subjects, WEALTHY gave a signal as good as the reference system in $82.6 \pm 4.1 \%$ of time for ECG and $88.4 \pm 1.5 \%$ of time for RESP;

2) This proportion goes up significantly when the subject is sweating. After several minutes of heavy sweating, the signal from WEALTHY is regularly better than the reference signal;

3) In cardiac patients, WEALTHY is not consistently different than a standard wireless recorder.

4) It is the feeling of the evaluators that the general performance of the WEALTHY system could be enhanced by a following post-processing as well as by a customisation of the WEALTHY garment to the subject/patient.

\section{Acknowledgements}

The authors are indebt to Drs G. Savourey, J.C. Launay and B. Melin for their technical skills during the validation phase in healthy subjects.

\section{References}

[1] R. Paradiso, "Tessuto in maglia per il monitoraggio di segnali vitali", Italian Patent N. FI2003A000308, December 3, 2003.

[2] G. Loriga, A. Scozzari, "Dispositivo elettronico indossabile per il monitoraggio di variabili fisiologiche attraverso misure di impedenza elettrica corporea", Italian Patent $\mathrm{N}$. PI/2004/A/000060, September 6, 2004

Address for correspondence:

Dr Rita Paradiso

SMARTEX, Via Giuntini, 13L, 56023 Navacchio (Pi), Italy. rita@smartex.it 